\title{
Granulomatous Reaction Confined to Two Blue-ink Tattoos after H1N1 Influenza Vaccine
}

Marc Z Handler $^{1 *}$, Viseslav Tonkovic-Capin ${ }^{2}$, Samuel D Brewster ${ }^{3}$, Thomas J Fritzlen ${ }^{2}$ and Daniel J Aires ${ }^{2}$

${ }^{1}$ Physician, University of Nebraska, 609 Cordova, Davis, CA 95616, USA

${ }^{2}$ University of Kansas Medical Center, 3901 Rainbow Blvd, Kansas City, KS 66160, USA

${ }^{3}$ Harrisonville Medical Clinic, 103 Oriole Street, Harrisonville, MO 64701, USA

\begin{abstract}
Noncaseating granulomas occur due to a hypersensitivity reaction to an exogenous antigen. The introduction of the H1N1 vaccine has been successful in disease prevention but we expand the current literature on possible adverse reactions to the vaccine to include granulomatous reactions.
\end{abstract}

\section{Report of a Case}

A 33-year-old white female presented to dermatology clinic with a three-month history of swelling and itching of blue-ink tattoos over each of her deltoid muscles. These symptoms began within one week of receiving intramuscular H1N1 vaccine (Sanofi-Pasteur, multidose vial containing $0.01 \%$ thimerosal, lot number UP005AA with expiration date March 15th 2011) into the right deltoid muscle superior to the tattoo on the right deltoid. These tattoos were placed eight years prior at different tattoo parlors. The patient denied past cutaneous eruptions or history of sarcoidosis or other vaccines or tattoos during this period. Past medical history included depression treated with depakote and haloperidol; hysterectomy with right oophorectomy and obesity. No medications had changed in the previous four months. Social history included occasional alcohol use but no tobacco or intravenous drug abuse. Physical examination showed induration confined to the blue-ink containing skin of two tattoos over the patient's deltoid muscles (Figure 1). Other tattoos containing black, red and yellow ink were not affected.

Histology of the punch biopsy specimen revealed noncaseating granulomas containing fine black granules (Figure 2). Staining revealed no evidence of acid-fast bacilli or fungi. Pathohistological differential diagnosis was sarcoidosis versus granulomatous reaction to pigment granules.

Workup for sarcoidosis included chest X-rays, pulmonary function testing, ophthalmological examination and angiotensin converting enzyme serum levels. All studies were within normal limits except for pulmonary function testing which revealed a moderate restrictive

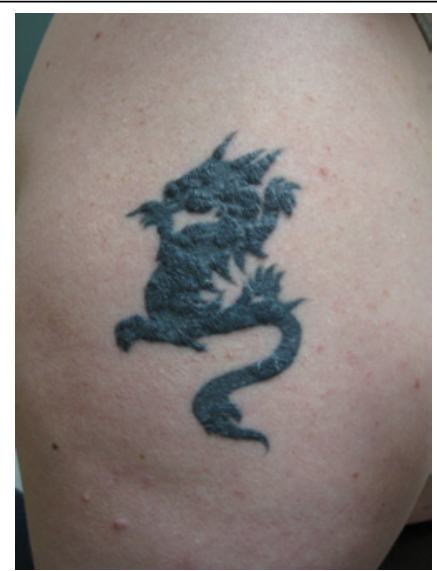

Figure 1: Edematous blue pigmented tattoo

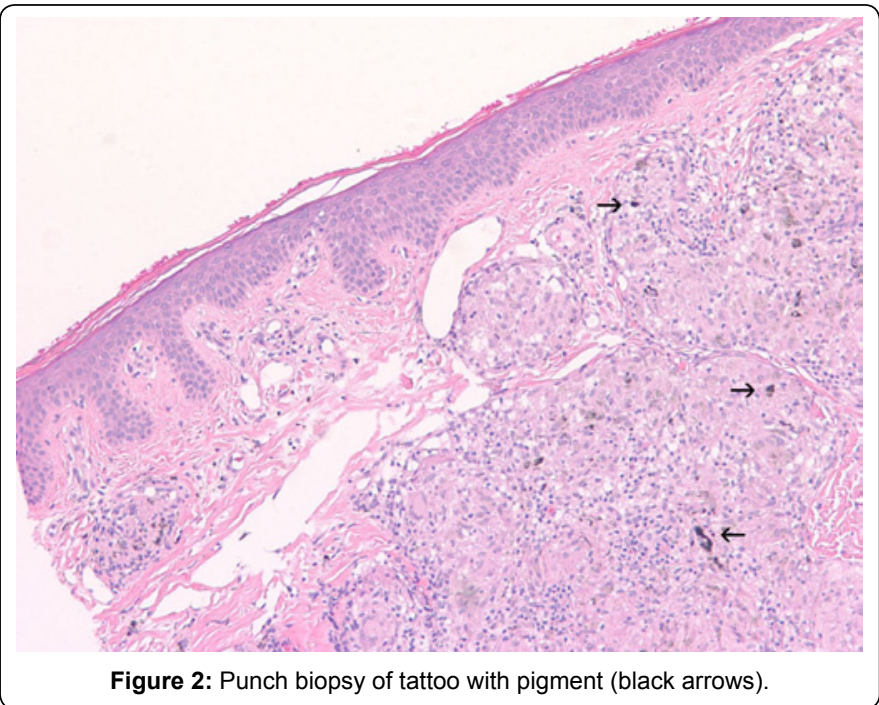

lung defect. The patient declined further workup, as well as referral to a pulmonologist. A diagnosis was made of granulomatous reaction confined to blue-ink tattoos possibly triggered by $\mathrm{H} 1 \mathrm{~N} 1$ vaccine. After failing a trial of clobetasol ointment twice daily the patient is currently well-controlled with intralesional injections of triamcinolone acetonide.

\section{Comment}

Noncaseating granulomas in patients with edema and pruritus of tattoos have been attributed to both sarcoidosis and delayed type hypersensitivity reactions. ${ }^{1}$ Sarcoidosis cannot be excluded even if foreign pigment is found in a biopsy site [1].

Sarcoidosis is a common disorder of the lungs and skin with

*Corresponding author: Marc Z Handler, Physician, University of Nebraska, 609 Cordova, Davis, CA 95616, USA, E-mail: mhandler@unmc.edu

Received November 14, 2010; Accepted December 15, 2010; Published December 19, 2010

Citation: Handler MZ, Tonkovic-Capin V, Brewster SD, Fritzlen TJ, Aires DJ (2010) Granulomatous Reaction Confined to Two Blue-ink Tattoos after H1N1 Influenza Vaccine. J Vaccines Vaccin 1: 108.doi:10.4172/2157-7560.1000108

Copyright: (c) 2010 Handler MZ, et al. This is an open-access article distributed under the terms of the Creative Commons Attribution License, which permits unrestricted use, distribution, and reproduction in any medium, provided the original author and source are credited. 
a prevalence of 6 per 100,000 per year [2]. Cutaneous sarcoidosis may present in conjunction with systemic findings or as the sole manifestation of disease. Multiple case reports have described sarcoidosis initially presenting as granulomas in single tattooed skin up to one year after placement $[1,3]$.

Medications and vaccines act as immune system modulators and have been linked to granulomatous reactions. IFN-alpha, TNFalpha blockers and BCG vaccine have been linked to sarcoidosis [4,5]. The administration of the H1N1 vaccine has not been previously documented to cause granulomatous reactions.

Another possible cause is the metal contained in the vaccine. Thimerosal preservative is a known allergen and has potential to be an immune system stimulator producing a granulomatous response. Further investigation is necessary regarding vaccination with and without thimerosal and cutaneous reactions.

The pathophysiology that confined the reaction to two blueink tattoos is unknown. One plausible explanation could be the release of tattoo pigment, containing cobalt, into extracellular space during immunization, triggering a granulomatous reaction. A similar mechanism was proposed for a 38-year-old man who developed a delayed type hypersensitivity reaction to red dye after red tattoo laser ablation [6]. The precise relationship, if any, between H1N1 vaccination and cutaneous granulomas remains unclear. Therefore, the validity of observation presented in this case report can only be confirmed by possible future reports of association of H1N1 vaccination with granulomatous tattoo reactions.

\section{References}

1. Morales-Callaghan AM, Aguilar-Bernier M, Martinez-Garcia G, MirandaRomero A (2006) Sarcoid granulomas on black tattoo. J Am Acad Dermatol 55: $S 71-73$

2. Nawras A, Alsolaiman MM, Mehboob S, Bartholomew C, Maliakkal B (2002) Systemic sarcoidosis presenting as a granulomatous tattoo reaction secondary to interferon- $\alpha$ treatment for chronic hepatitis $C$ and review of the literature. Dig Dis Sci 47: 1627-1631.

3. Yoong C, Vun, YY, Spelman L, Muir J (2010) True blue football fan: Tattoo reaction confined to blue pigment. Australas J Dermatol 51: 21-22.

4. Daien $\mathrm{Cl}$, Monnier A, Claudepierre P, Constantin A, Eschard JP et al. (2000) Sarcoid-like granulomatosis in patients treated with tumor necrosis factor blockers: 10 cases. Rheumatology 48: 883-886.

5. Osborne GE, Mallon E, Mayou SC (2003) Juvenile sarcoidosis after BCG vaccination. J Am Acad Dermatol 48: S99-102.

6. Bhardwaj SS, Brodell RT, Taylor JS (2003) Red tattoo reactions. Contact Dermatitis 48: 236-237. 